NSF-KITP-11-098

\title{
The QCD trace anomaly
}

\author{
Jens O. Andersen, ${ }^{1}$ Lars E. Leganger, ${ }^{1}$ Michael Strickland, ${ }^{2,3,4}$ and $\mathrm{Nan} \mathrm{Su}^{5,1}$ \\ ${ }^{1}$ Department of Physics, Norwegian University of \\ Science and Technology, N-7491 Trondheim, Norway \\ ${ }^{2}$ Department of Physics, Gettysburg College, \\ Gettysburg, Pennsylvania 17325, USA \\ ${ }^{3}$ Kavli Institute for Theoretical Physics, \\ University of California, Santa Barbara, CA 93117 \\ ${ }^{4}$ Frankfurt Institute for Advanced Studies, \\ D-60438 Frankfurt am Main, Germany \\ ${ }^{5}$ Faculty of Physics, Bielefeld University, D-33615 Bielefeld, Germany
}

\begin{abstract}
In this brief report we compare the predictions of a recent next-to-next-to-leading order hardthermal-loop perturbation theory (HTLpt) calculation of the QCD trace anomaly to available lattice data. We focus on the trace anomaly scaled by $T^{2}$ in two cases: $N_{f}=0$ and $N_{f}=3$. When using the canonical value of $\mu=2 \pi T$ for the renormalization scale, we find that for YangMills theory $\left(N_{f}=0\right)$ agreement between HTLpt and lattice data for the $T^{2}$-scaled trace anomaly begins at temperatures on the order of $8 T_{c}$ while when including quarks $\left(N_{f}=3\right)$ agreement begins already at temperatures above $2 T_{c}$. In both cases we find that at very high temperatures the $T^{2}$-scaled trace anomaly increases with temperature in accordance with the predictions of HTLpt.
\end{abstract}

PACS numbers: 11.15.Bt, 04.25.Nx, 11.10.Wx, 12.38.Mh 
InTRODUCTION : The QCD equation of state (EoS) is of crucial importance for a better understanding of the matter created in the ultrarelativistic heavy-ion collisions [1], as well as the candidates for dark matter in cosmology [2]. Due to the nonperturbative nature of QCD at low temperatures and small chemical potentials where the system is strongly coupled, lattice gauge theory is by far the most successful method for determining the EoS in this part of the phase diagram. However, due to the asymptotic freedom of QCD, lattice calculations should be compatible with perturbation theory at very high temperatures where the coupling constant becomes asymptotically weak.

The calculation of QCD thermodynamics utilizing weakly-coupled quantum field theory has a long history [3 5]. The free energy is now known up to order $g_{s}^{6} \log g_{s}$ [5]. Unfortunately, a straightforward application of perturbation theory is of no quantitative use at the phenomenology-relevant intermediate coupling regime which is probed in the ongoing ultrarelativistic heavy-ion collision experiments at Brookhaven National Laboratory's Relativistic Heavy Ion Collider and the European Organization for Nuclear Research's Large Hadron Collider (LHC). The issue is that the weak-coupling expansion series oscillates wildly and shows no sign of convergence unless the temperature is astronomically high. For example, simply comparing the magnitude of low-order contributions to the QCD free energy with three quark flavors $\left(N_{f}=3\right)$, one finds that the $g_{s}^{3}$ contribution is smaller than the $g_{s}^{2}$ contribution only for $g_{s} \lesssim 0.9$ or $\alpha_{s} \lesssim 0.07$ which corresponds to a temperature of $T \sim 10^{5} \mathrm{GeV}$ $\sim 5 \times 10^{5} T_{c}$, with $T_{c} \sim 170 \mathrm{MeV}$ being the QCD critical temperature.

The poor convergence of the weak-coupling expansion of thermodynamic functions stems from the fact that at high temperature the classical solution is not described by massless particles, but instead massive quasiparticles due to plasma effects such as the screening of (chromo)electric fields and Landau damping. One way to deal with the problem is to use an effective field theory framework in which one treats hard modes using standard four-dimensional QCD and soft modes using a dimensionally reduced three-dimensional $\mathrm{SU}(3)$ plus adjoint Higgs model [5/7] but treating the soft sector non-perturbatively by not expanding the soft contribution in powers of the coupling constant [8]. This picture of treating the soft sector non-perturbatively is ubiquitous and there exist several ways of systematically reorganizing the perturbative series at finite temperature [9]. Such treatments are based on a quasiparticle picture in which one performs a loop expansion around an ideal gas of massive quasiparticles, rather than an ideal gas of massless particles. In this 
brief report, we will present results for the trace anomaly in pure Yang-Mills $\left(N_{f}=0\right)$ and QCD with $N_{f}=3$ obtained using the hard-thermal-loop perturbation theory (HTLpt) reorganization [10 12]. We will focus on the quantity $(\mathcal{E}-3 \mathcal{P}) / T^{2}$ which, in pure Yang-Mills, has been shown to be approximately constant for temperatures which are close to the phase transition temperature. We will show that, when using the trace anomaly scaled by $T^{2}$, $(\mathcal{E}-3 \mathcal{P}) / T^{2}$, as a criterion, pure Yang-Mills exhibits a transition to perturbative behavior at temperatures on the order of $8 T_{c}$ while for $N_{f}=3$ one sees reasonable agreement with the next-to-next-to-leading order (NNLO) HTLpt result already at temperatures on the order of $2-3 T_{c}$.

BACKGROUND : HTLpt is a reorganization of the perturbative series for thermal gauge theory that is a gauge-invariant generalization of screened perturbation theory (SPT) applied to scalar $\phi^{4}$ theory [13]. In scalar $\phi^{4}$ theory the basic idea of SPT is to add and subtract a thermal mass term from the bare Lagrangian: including the added piece in the free part of the Lagrangian; while treating the subtracted piece as an interaction term on the same footing as the quartic term. In gauge theories, however, simply adding and subtracting a local mass term violates gauge invariance [14]. Instead, one adds and subtracts the hard-thermal-loop (HTL) effective action, which dresses the propagators and vertices self-consistently so that the reorganization is manifestly gauge invariant [15]. HTLpt thermodynamic calculations for non-Abelian theories have been recently carried out to three loops, aka NNLO [11, 12] and improved convergence comparing to the weak-coupling expansion is found.

At temperatures just above the phase transition, $1.1 T_{c} \lesssim T \lesssim 4 T_{c}$, in pure Yang-Mills, lattice results find that the trace anomaly scaled by $T^{2}$ is approximately constant. It has been suggested that this behavior is due to the influence of power corrections of order $T^{2}$ [16] which are nonperturbative in nature and might be related to confinement. Phenomenological fits of lattice data which include such power corrections show that the agreement with lattice data is improved [17]. Alternatively, the power corrections are obtained from AdS/QCD models which break conformal invariance [18 21]. The potential impact of the power corrections on relativistic hydrodynamics relevant to heavy ion experiments at LHC is explored in Ref. [22]. However, it is important to mention that current AdS/QCD models have no firm theoretical reason for including modifications which induce power law corrections besides their seeming phenomenological importance. In addition, the models are largely unconstrained in the 


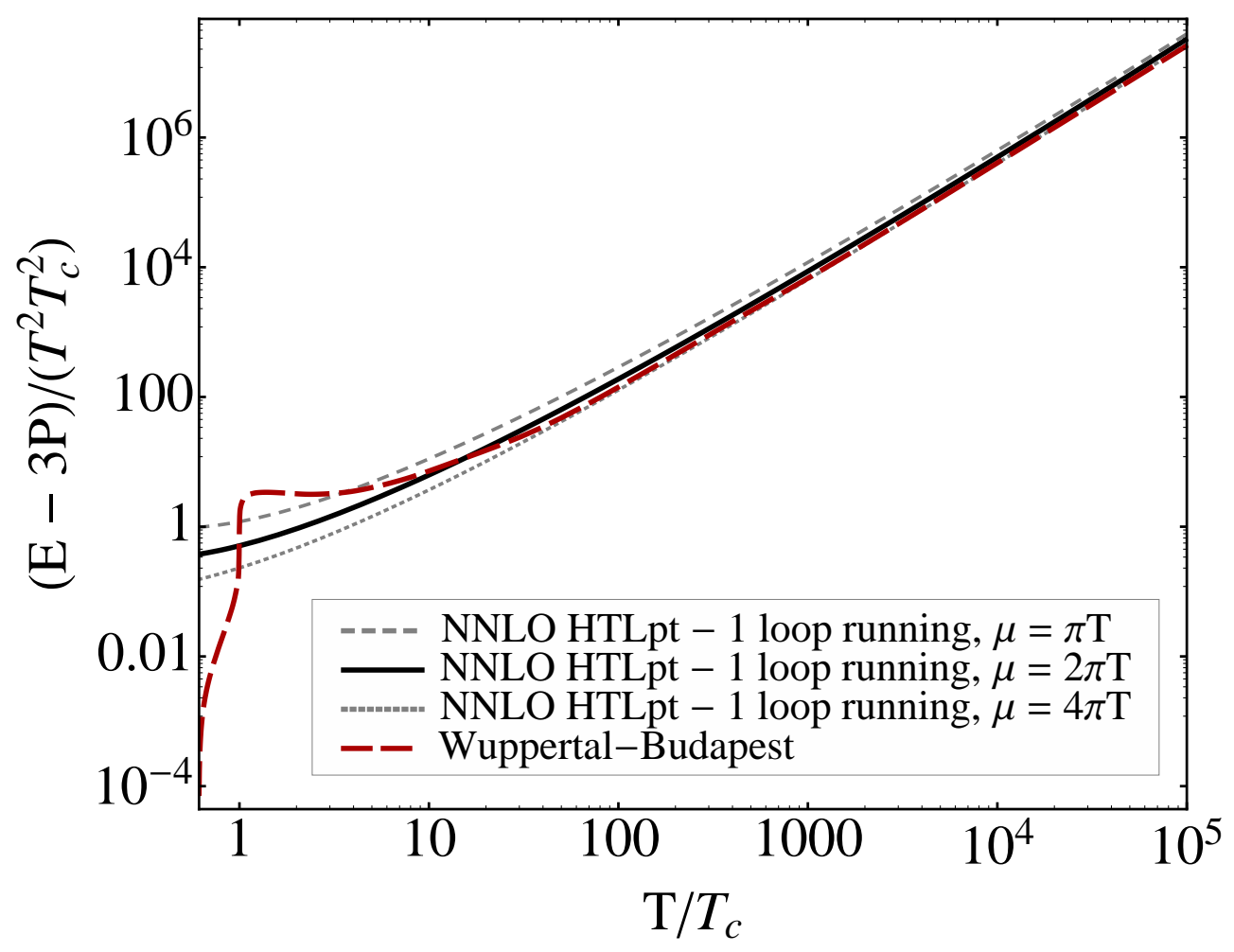

FIG. 1. NNLO HTLpt result for the pure Yang-Mills $\left(N_{f}=0\right)$ trace anomaly over temperature squared compared with lattice data from the Wuppertal-Budapest group [24] at high temperatures. For all curves we have used the one-loop running of the strong coupling as specified in the text. Different HTLpt curves correspond to different choices of the renormalization scale $\mu$.

magnitude of these corrections and the parameters in the underlying models must be fit to lattice data.

In the last decade there have been intensive studies of the EoS on the lattice for both pure Yang-Mills and full QCD with dynamical quarks. These studies generally measure the trace anomaly scaled by $T^{4},(\mathcal{E}-3 \mathcal{P}) / T^{4}$, and use this to determine the energy density, pressure, and entropy density of the system. In the remainder of this brief report, we compare the NNLO HTLpt trace anomaly with lattice data from the Wuppertal-Budapest [24, 25], HotQCD [26, 27], RBC-Bielefeld [28, 29], and WHOT-QCD [30, 31] collaborations.

RESUlTs : In Fig. 1 we plot the trace anomaly scaled by $T^{2} T_{c}^{2}$ for pure Yang-Mills theory and compare this with recent lattice results obtained by the Wuppertal-Budapest collaboration [24]. This figure shows the behavior of $\Delta \equiv(\mathcal{E}-3 \mathcal{P}) /\left(T^{2} T_{c}^{2}\right)$ at high temperatures. 


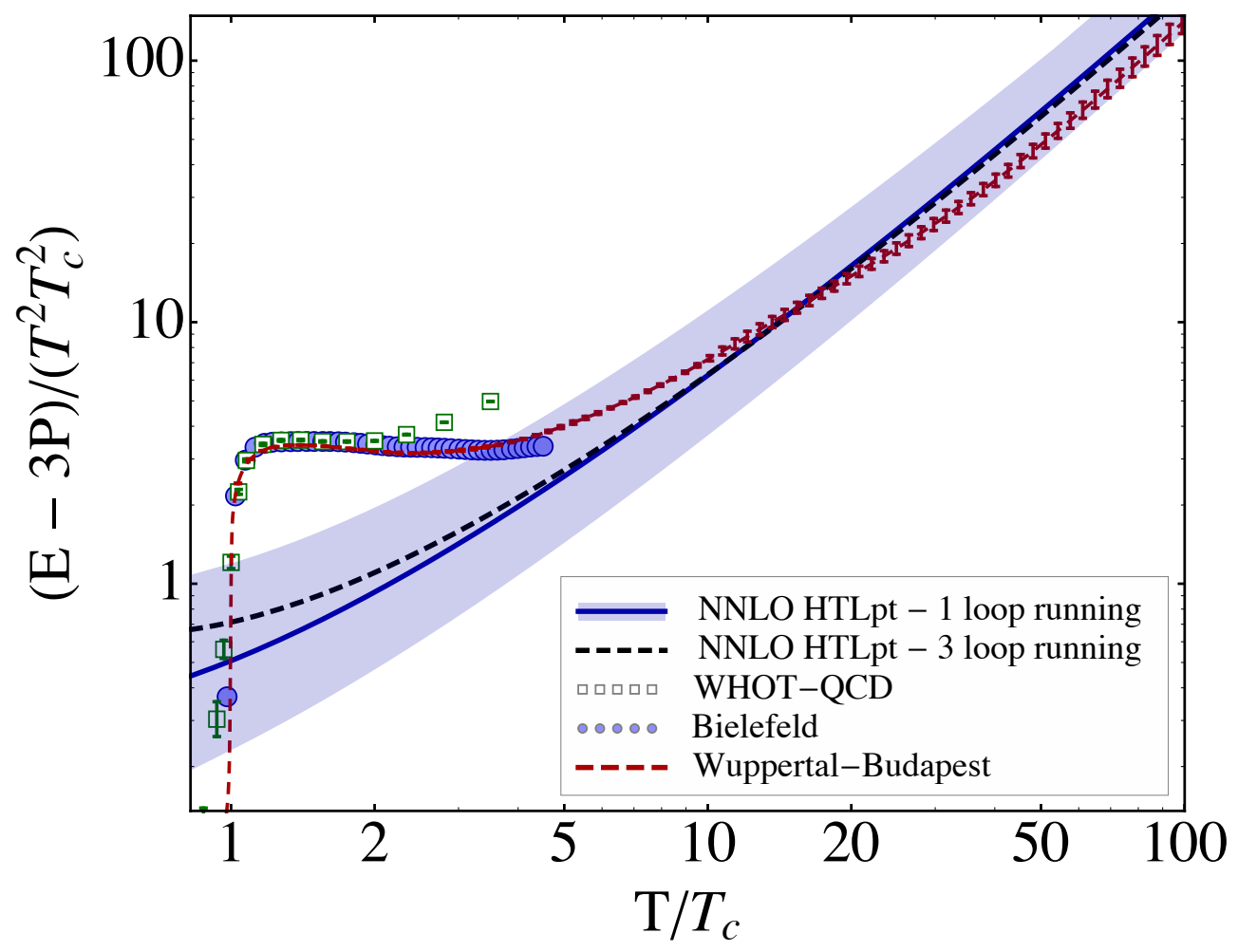

FIG. 2. NNLO HTLpt result for the pure Yang-Mills $\left(N_{f}=0\right)$ trace anomaly over temperature squared compared with lattice data from the Wuppertal-Budapest [24], Bielefeld [23], and WHOT-QCD [30, 31] collaborations. For the NNLO HTLpt result with three-loop running, the renormalization scale was taken to be $\mu=2 \pi T$. For the NNLO HLTpt result with one-loop running, we show a line corresponding to $\mu=2 \pi T$ and a band showing the range $\pi T \leq \mu \leq 4 \pi T$.

For the NNLO HTLpt result we use the perturbative one-loop running of the strong coupling which is consistent with the counterterms that one obtains at NNLO. We show three different curves corresponding to the three different values for the renormalization scale, $\mu=\{\pi T, 2 \pi T, 4 \pi T\}$. The central value of $\mu=2 \pi T$ is the scale one expects to emerge from an equilibrium calculation since the lowest possible non-vanishing bosonic Matsubara mode sets the scale; however, for completeness sake we show the variation of $\mu$ by a factor of two in either direction, which should be understood as a dramatic overestimate of the theoretical uncertainty. As can be seen from Fig. 1, at low temperatures NNLO HTLpt underpredicts the trace anomaly for pure Yang-Mills and only starts to agree at temperatures on the order of $8 T_{c}$ for $\mu=2 \pi T$. The agreement with lattice results begins at much higher temperatures 
for the other extreme values of $\mu$. At asymptotically high temperatures we see excellent agreement between the NNLO HTLpt results and the lattice results. We note in this context that if one allows for a fit to the unknown perturbative $g^{6}$ contribution to the pressure, then the EQCD approach can equally-well describe the pure Yang-Mills lattice data down to temperatures on the order of $8 T_{c}$ [6, 7]. In the EQCD case, once again, the key is to not expand perturbatively in the Debye mass, but to instead treat contributions from this scale non-perturbatively.

The behavior of the trace anomaly at lower temperatures is presented in Fig. 2 along with lattice data from the Bielefeld [23] and WHOT-QCD [30, 31] collaborations. The solid black line is the NNLO HTLpt result obtained using a one-loop running of the QCD coupling and the dashed black line is the HTLpt result obtained using a three-loop running of the QCD coupling. In the case of the three-loop running we use the lattice determination of $T_{c} / \Lambda_{\overline{\mathrm{MS}}}=1.26$ to fix the scale [24]. For comparison between the one- and three-loop results we require that both give the same value for the strong coupling when the renormalization scale $\mu=5 \mathrm{GeV}$. Numerically, one finds $\alpha_{s}(5 \mathrm{GeV})=0.140553$. We compare one- and three-loop running because, formally speaking, one only obtains counterterms consistent with one-loop running from the NNLO HTLpt calculation; however, at temperatures close to the critical temperature the three-loop running gives important corrections to the scale dependence of the running coupling [32]. We will use the difference between one- and threeloop running to gauge the theoretical uncertainty of the NNLO HTLpt results. For both the one- and three-loop running the lines assume $\mu=2 \pi T$ to fix the renormalization scale as a function of the temperature. For the one-loop running case we also show a band corresponding to varying the renormalization scale in the range $\pi T \leq \mu \leq 4 \pi T$. We note that this value is not fit in any way to the lattice data, but instead is the predicted value of the appropriate renormalization scale to use. Better agreement at high temperatures can be obtained by fitting $\mu$ to lattice data, in which case one finds that a value of $\mu /(2 \pi T)=1.75$ is preferred in the case of pure Yang-Mills [24]. Here we present the results at face value without fitting.

The most remarkable feature of the lattice data for $\Delta$ is that it is essentially constant in the temperature range $T \sim 1.1-4 T_{c}$. At temperatures above approximately $4 T_{c}$ the latest Wuppertal-Budapest [24, 25] results show an upward trend in accordance with perturbative predictions. The WHOT-QCD results also exhibit an upward trend, however, it starts at 


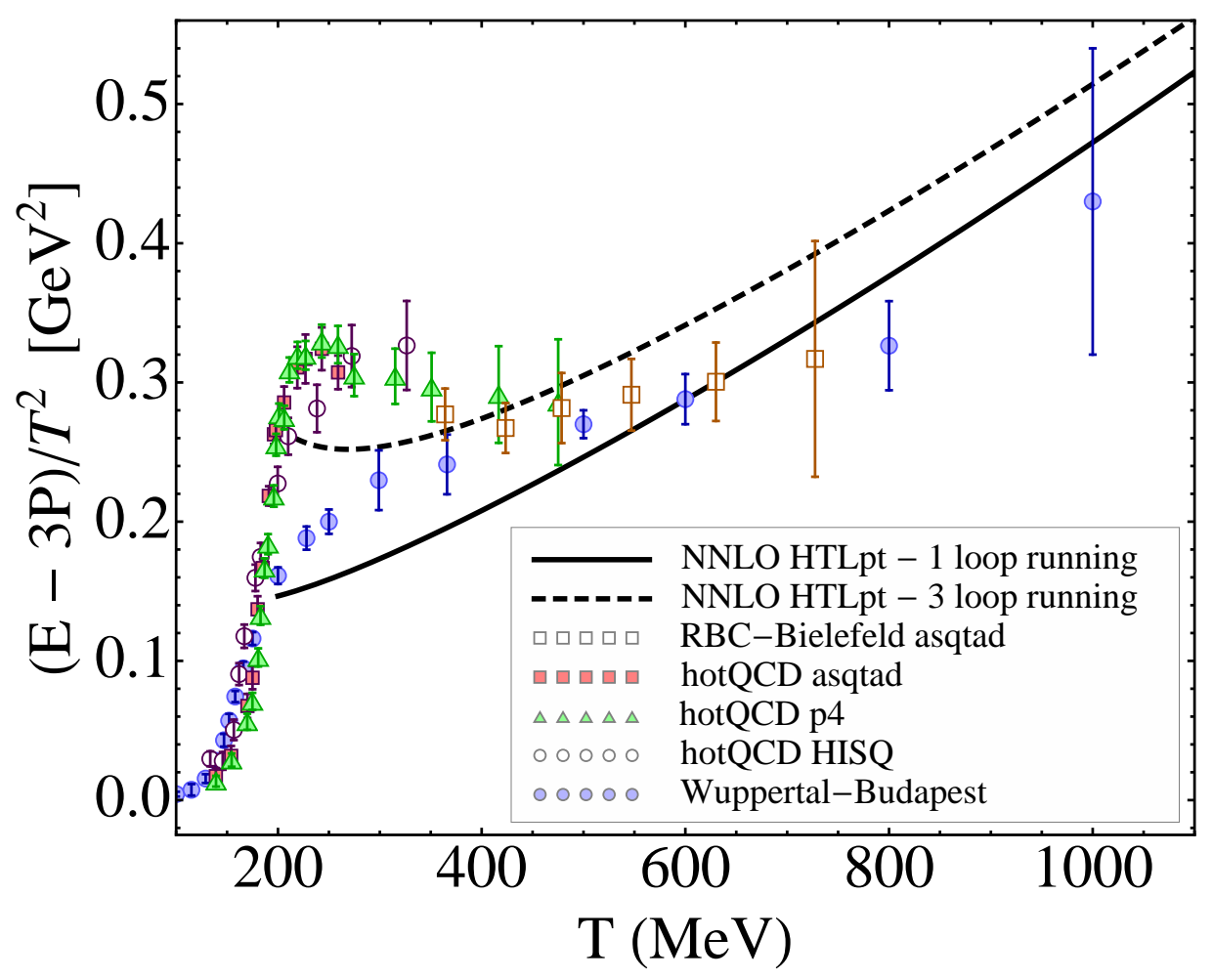

FIG. 3. NNLO HTLpt result for the $N_{f}=3$ QCD trace anomaly over temperature squared compared with lattice data from the Wuppertal-Budapest [25], hotQCD [26, 27], and RBC-Bielefeld [28, 29] collaborations. For both HTLpt curves the renormalization scale was taken to be $\mu=2 \pi T$.

much lower temperatures. This discrepancy could be due to their fixed scale approach not having sufficiently large $N_{\tau}$ at high temperatures as noted in their paper [30].

In Fig. 3 we present the $N_{f}=3$ QCD NNLO HTLpt prediction for $(\mathcal{E}-3 \mathcal{P}) / T^{2}$ and compare to lattice results available from the Wuppertal-Budapest [25] and hotQCD collaborations [26, 27]. The lattice data from the Wuppertal-Budapest collaboration uses the stout action and have been continuum estimated by averaging the trace anomaly measured using their two smallest lattice spacings corresponding to $N_{\tau}=8$ and $N_{\tau}=10$. The lattice data from the hotQCD collaboration are their $N_{\tau}=8$ results using the asqtad, p4, and HISQ actions which have not been continuum extrapolated [26, 27]. The lattice data from the RBC-Bielefeld collaboration is $N_{\tau}=6$ and have also not been continuum extrapolated [28, 29].

As before, we present HTLpt results using both one- and three-loop running of the 


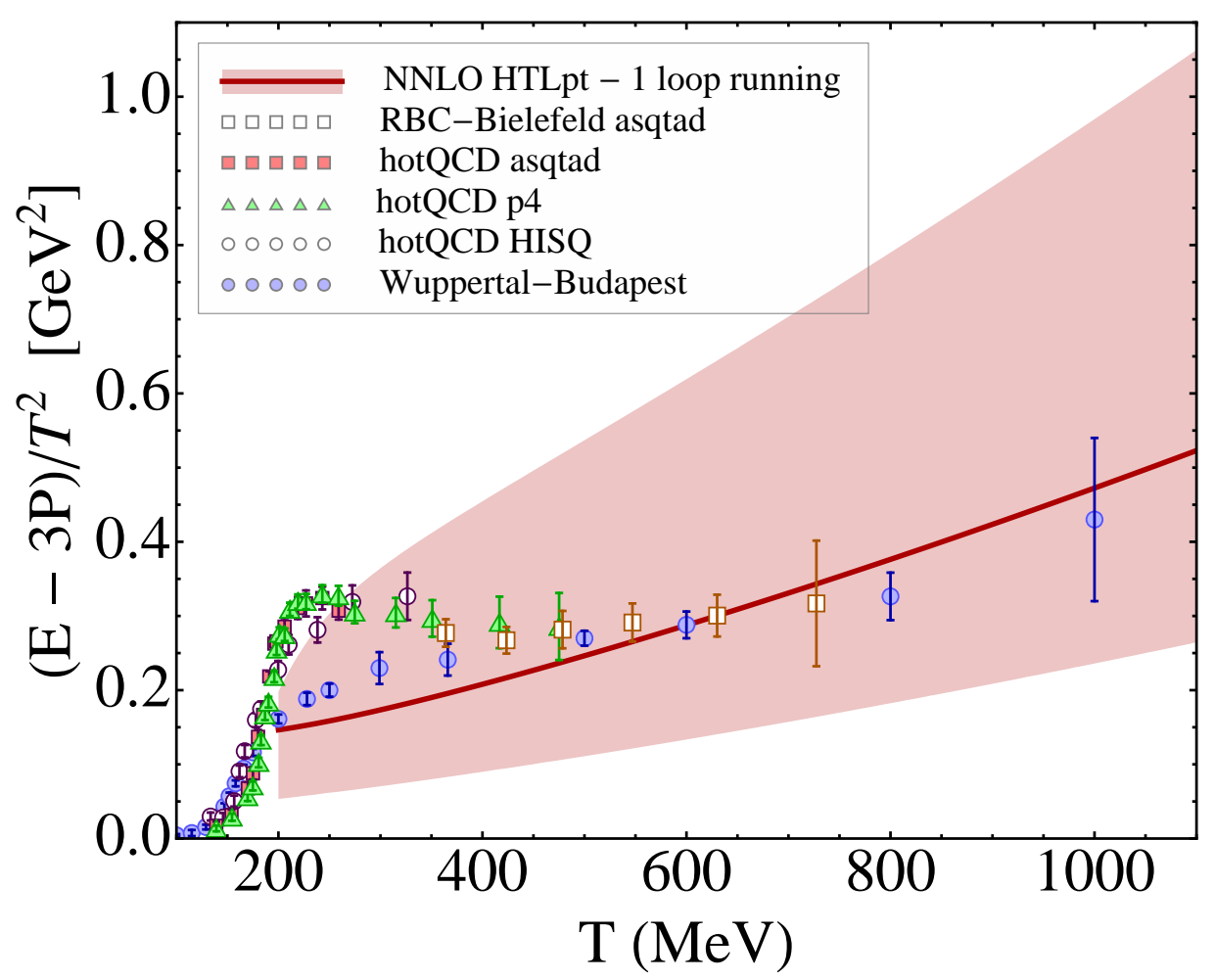

FIG. 4. NNLO HTLpt result for the $N_{f}=3$ QCD trace anomaly over temperature squared compared with lattice data from the Wuppertal-Budapest [25], hotQCD [26, 27], and RBC-Bielefeld [28, 29] collaborations. For the HTLpt curve we use the one-loop running of the strong coupling constant. The red band is the variation of the HTLpt result with the assumed renormalization group scale $(\pi T \leq \mu \leq 4 \pi T)$ and the line corresponds to the value $\mu=2 \pi T$.

strong coupling. In this case we have required $\alpha_{s}(5 \mathrm{GeV})=0.2034$ in accordance with recent high precision lattice measurements of the running coupling constant [33]. As can be seen from Fig. 3 for $T>400 \mathrm{MeV}$ we find reasonable agreement between the NNLO HTLpt predictions and available lattice results. Below this temperature the WuppertalBudapest and hotQCD results do not seem to agree. Therefore, it is difficult to draw conclusions about the efficacy of the HTLpt approach; however, naively one expects that for temperatures less than twice the critical temperature that non-perturbative corrections should become increasingly important.

In Fig. 4 we again present the $N_{f}=3$ QCD NNLO HTLpt prediction for $(\mathcal{E}-3 \mathcal{P}) / T^{2}$ and compare to lattice results. However, in this case we show only the result of assuming one-loop 
running for the strong coupling constant which is consistent with the counterterms necessary to renormalize HTLpt at NNLO. The red band results from varying the renormalization scale $\mu$ by a factor of two around $\mu=2 \pi T$ which is the theoretically favored value. As can be seen from this figure, it would seem at first glance that there is still a significant theoretical uncertainty related to the choice of the renormalization scale $\mu$; however, $\mu$ is not a completely free parameter since its value should be related to the lowest possible momentum exchange, which for a plasma in equilibrium is set by the lowest non-vanishing bosonic Matsubara mode. In this sense, the band shown dramatically overestimates the theoretical error of the final result; however, we include it here for completeness.

Discussion And Conclusion: The purpose of this brief report is to present an easily accessible comparison of predictions of the $T^{2}$-scaled trace anomaly with recent NNLO calculations using HTLpt. The details of the HTLpt framework and the full NNLO calculation itself can be found in Refs. [11, 12]. We compared the $N_{c}=3$ NNLO HTLpt predictions with the QCD $T^{2}$-scaled trace anomaly in two specific cases, namely $N_{f}=0$ and $N_{f}=3$, however, the result for general $N_{c}$ and $N_{f}$ can be found in Refs. [11, 12.

We find that by including quarks in the calculation, agreement with lattice data is greatly improved as compared to the NNLO results of pure-glue QCD. Fermions are perturbative in the sense that they decouple in the dimensional-reduction step of effective field theory, so we expect that including contributions from quarks gives at least as good agreement with the lattice calculations as compared to the pure-glue case. However, the reasons for the large improvement between the HTLpt predictions and the lattice calculations is not clear to us.

The $T^{2}$-scaling of the trace anomaly has received much attention recently and comparisons with lattice data provides an extremely sensitive test of the agreement between theory and numerical results. Using $\mu=2 \pi T$ we find that for Yang-Mills theory $\left(N_{f}=0\right)$ agreement between HTLpt and lattice data for the trace anomaly begins at temperatures on the order of $8 T_{c}$ while when including quarks $\left(N_{f}=3\right)$ the agreement begins already at temperatures above $2 T_{c}$. In both cases we find that at very high temperatures the $T^{2}$-scaled trace anomaly increases with temperature in accordance with the predictions of HTLpt.

In closing we emphasize again that the $T^{2}$-scaled trace anomaly is a very sensitive observable. If one were to compare instead the pressure, energy density, and entropy density one finds that, in both cases presented here, HTLpt is consistent with lattice data down 
to temperatures on the order of $2-3 T_{c}$ [11, 12]. Looking forward, we point out that the HTLpt framework is formulated in Minkowski space and is therefore also applicable to the calculation of real-time properties of the quark gluon plasma at LHC temperatures.

ACKnowledgments : The authors would like to thank A. Dumitru for encouraging us to summarize our results in this form. We thank the Wuppertal-Budapest, hotQCD, and RBCBielefeld collaborations for providing their lattice data. M. S. was supported in part by the Helmholtz International Center for FAIR Landesoffensive zur Entwicklung WissenschaftlichÖkonomischer Exzellenz program and the Kavli Institute for Theoretical Physics grant No. NSF PHY05-51164. N. S. acknowledges support from the Yggdrasil mobility program of the Norwegian Research Council, the Sofja Kovalevskaja program of the Alexander von Humboldt Foundation, and the kind hospitality of the Department of Physics at the Norwegian University of Science and Technology.

[1] I. Arsene et al. (BRAHMS Collaboration), Nucl. Phys. A757, 1 (2005); B. B. Back et al. (PHOBOS Collaboration), Nucl. Phys. A757, 28 (2005); J. Adams et al. (STAR Collaboration), Nucl. Phys. A757, 102 (2005); K. Adcox et al. (PHENIX Collaboration), Nucl. Phys. A757, 184 (2005); M. Gyulassy and L. McLerran, Nucl. Phys. A750, 30 (2005).

[2] M. Hindmarsh and O. Philipsen, Phys. Rev. D 71, 087302 (2005); M. Laine, Proc. Sci., LAT2006 (2006) 014.

[3] E. V. Shuryak, Sov. Phys. JETP 47, 212 (1978) [Zh. Eksp. Teor. Fiz. 74, 408 (1978)]; J. I. Kapusta, Nucl. Phys. B148 461-498 (1979). T. Toimela, Int. J. Theor. Phys. 24, 901 (1985); 26, 1021(E) (1987); P. Arnold and C. X. Zhai, Phys. Rev. D 50, 7603 (1994); Phys. Rev. D 51, 1906 (1995); C. X. Zhai and B. Kastening, Phys. Rev. D 52, 7232 (1995).

[4] E. Braaten and A. Nieto, Phys. Rev. Lett. 76, 1417 (1996); Phys. Rev. D 53, 3421 (1996).

[5] K. Kajantie, M. Laine, K. Rummukainen and Y. Schröder, Phys. Rev. D 67, 105008 (2003).

[6] A. Hietanen, K. Kajantie, M. Laine, K. Rummukainen and Y. Schroder, Phys. Rev. D 79, 045018 (2009).

[7] M. Laine and Y. Schroder, Phys. Rev. D 73, 085009 (2006) [arXiv:hep-ph/0603048].

[8] J. P. Blaizot, E. Iancu, A. Rebhan, Phys. Rev. D68, 025011 (2003). [hep-ph/0303045].

[9] J. P. Blaizot, E. Iancu and A. Rebhan, arXiv:hep-ph/0303185; U. Kraemmer and A. Rebhan, 
Rep. Prog. Phys. 67, 351 (2004); J. O. Andersen and M. Strickland, Ann. Phys. (N.Y.) 317, $281(2005)$.

[10] J. O. Andersen, E. Braaten, E. Petitgirard and M. Strickland, Phys. Rev. D 66, 085016 (2002);

J. O. Andersen, E. Petitgirard, and M. Strickland, Phys. Rev. D 70, 045001 (2004).

[11] J. O. Andersen, M. Strickland and N. Su, Phys. Rev. Lett. 104, 122003 (2010); J. High Energy Phys. 08 (2010) 113.

[12] J. O. Andersen, L. E. Leganger, M. Strickland and N. Su, Phys. Lett. B 696, 468 (2011); arXiv:1103.2528 [hep-ph].

[13] F. Karsch, A. Patkos and P. Petreczky, Phys. Lett. B 401, 69 (1997); S. Chiku and T. Hatsuda, Phys. Rev. D 58, 076001 (1998); J. O. Andersen, E. Braaten and M. Strickland, Phys. Rev. D 63, 105008 (2001).

[14] W. Buchmüller and O. Philipsen, Nucl. Phys. B443, 47 (1995); G. Alexanian and V. P. Nair, Phys. Lett. B 352, 435 (1995).

[15] E. Braaten and R. D. Pisarski, Phys. Rev. D 45, R1827 (1992).

[16] K. I. Kondo, Phys. Lett. B 514, 335 (2001); P. N. Meisinger, T. R. Miller and M. C. Ogilvie, Phys. Rev. D 65, 034009 (2002); P. N. Meisinger, M. C. Ogilvie and T. R. Miller, Phys. Lett. B 585, 149 (2004); R. D. Pisarski, Phys. Rev. D 74, R121703 (2006); [Prog. Theor. Phys. Suppl. 168, 276 (2007); S. Narison and V. I. Zakharov, Phys. Lett. B 679, 355 (2009).

[17] E. Megias, E. Ruiz Arriola and L. L. Salcedo, Phys. Rev. D 80, 056005 (2009); Phys. Rev. D 81, 096009 (2010).

[18] O. Andreev, Phys. Rev. D 76, 087702 (2007); Phys. Rev. Lett. 102, 212001 (2009).

[19] S. S. Gubser, A. Nellore, Phys. Rev. D78, 086007 (2008).

[20] S. S. Gubser, A. Nellore, S. S. Pufu, F. D. Rocha, Phys. Rev. Lett. 101, 131601 (2008).

[21] J. Noronha, Phys. Rev. D81, 045011 (2010).

[22] O. Andreev, arXiv:1101.5481 [hep-ph].

[23] G. Boyd, J. Engels, F. Karsch, E. Laermann, C. Legeland, M. Lutgemeier and B. Petersson, Nucl. Phys. B469, 419 (1996).

[24] Sz. Borsanyi, G. Endrodi, Z. Fodor, S. D. Katz and K. K. Szabo, arXiv:1104.0013 [hep-ph].

[25] Sz. Borsanyi, G. Endrodi, Z. Fodor, A. Jakovac, S. D. Katz, S. Krieg, C. Ratti and K. K. Szabo, J. High Energy Phys. 11 (2010) 077.

[26] A. Bazavov et al., Phys. Rev. D 80014504 (2009). 
[27] A. Bazavov, P. Petreczky, PoS LATTICE2010, 169 (2010).

[28] M. Cheng, N. H. Christ, S. Datta, J. van der Heide, C. Jung, F. Karsch, O. Kaczmarek, E. Laermann et al., Phys. Rev. D77, 014511 (2008). [arXiv:0710.0354 [hep-lat]].

[29] P. Petreczky, Nucl. Phys. A830, 11C-18C (2009). [arXiv:0908.1917 [hep-ph]].

[30] T. Umeda, S. Ejiri, S. Aoki, T. Hatsuda, K. Kanaya, Y. Maezawa and H. Ohno (WHOT-QCD Collaboration), Phys. Rev. D 79051501 (2009).

[31] T. Umeda, S. Aoki, K. Kanaya, H. Ohno, S. Ejiri, T. Hatsuda and Y. Maezawa (WHOT-QCD Collaboration), Proc. Sci., LAT2010 (2010) 218.

[32] K. Nakamura et al. (Particle Data Group), J. Phys. G 37, 075021 (2010).

[33] C. McNeile, C. T. H. Davies, E. Follana, K. Hornbostel and G. P. Lepage, Phys. Rev. D 82, $034512(2010)$. 